\title{
Actualizaciones en la detección precoz de la osteoporosis
}

\section{Actualization in early detection of osteoporosis}

\author{
Corcuera Flores JR*, Manso Platero FJ*, Martínez Maestre MA***, \\ Machuca Portillo G**
}

\section{RESUMEN}

La osteoporosis es una enfermedad sistémica del esqueleto, con amplia prevalencia sobre todo en mujeres postmenopáusicas. La ortopantomografía (OPMG) es una técnica radiológica de uso generalizado en las consultas de los odontólogos. En el presente estudio se hace una revisión de trabajos en los que, utilizando una serie de índices como: Mandibular Cortical Index (MCI), Mental Index (MI), Panoramic Mandibular Index (PMI), Antegonial Index (AI), Gonial Index (GI), medidos sobre OMPG se plantea la hipótesis de poder detectar precozmente la osteoporosis por este medio.

Los resultados expuestos en los diferentes estudios avalan esta hipótesis y así se podrá remitir a los pacientes al especialista para que se les haga un estudio más exhaustivo sobre dicha patología.

Palabras clave: Osteoporosis, ortopantomografía, Index.

\section{SUMMARY}

Osteoporosis is a systemic skeletal disease, with widespread prevalence especially in postmenopausal women. Orthopantomography (OPMG) is a widely used diagnosis technique in dental practice. The objective of this study is to make a systematic review of studies in which, using a series of indices as Mandibular Cortical Index (MCI), Mental Index (MI), Panoramic Mandibular Index (PMI), Antegonial Index (AI), Gonial Index (GI) measured on panoramic radiographs are useful for detecting early osteoporosis.

The results reported in different studies support the possibility that OPMG could be an effective tool to detect early Osteoporosis and refer patients to a specialist to treat their disease.

Key words: Osteoporosis, orthopantomography, Index.

Fecha de recepción: 7 de septiembre de 2012.

Aceptado para publicación: 12 de noviembre de 2012.

* Profesor Asociado. Facultad de Odontología. Universidad de Sevilla (jcorcuera@us.es).

** Profesor Titular. Facultad de Odontología. Universidad de Sevilla (gmachuca@us.es).

*** Jefa de Sección. Hospital de la Mujer. Hospital Universitario "Virgen del Rocío". Sevilla.

Corcuera Flores JR, Manso Platero FJ, Martínez Maestre MA, Machuca Portillo G. Actualizaciones en la detección precoz de la osteoporosis. Av. Odontoestomatol 2014; 30 (5): 251-261.

\section{INTRODUCCIÓN}

El envejecimiento progresivo de las poblaciones en las sociedades occidentales ha llevado a un sector cada vez mayor de la población femenina a este rango de edades. No sorprende, por tanto, que la osteoporosis postmenopáusica sea un problema que haya alcanzado proporciones epidémicas y que sus notables repercusiones, unido a su frecuencia (en un imparable crescendo), la sitúen como problema de salud pública de primera magnitud. 


\section{La menopausia}

La menopausia se define como el cese definitivo de la menstruación, proceso fisiológico que se produce como consecuencia del fin de la actividad ovárica. Actualmente se estima que en los países occidentales, más de un $30 \%$ de las mujeres son postmenopáusicas.

\section{Menopausia y osteoporosis}

De toda la sintomatología referida a la menopausia, queremos destacar la osteoporosis, descrita por Albright y cols. (1).

La menopausia supone la condición de riesgo más importante para la Osteoporosis (2). En el mundo occidental se mantiene la edad media de presentación de la menopausia a los 49 años mientras que, la esperanza de vida ha aumentado hasta superar los 80 años. Esto condiciona que la mujer pase más de la tercera parte de su vida en menopausia. Esta circunstancia justifica que la prevalencia de la Osteoporosis haya aumentado de forma notable en los últimos años. La deprivación estrogénica, junto con el hecho de que el pico de masa ósea en la mujer es más precoz y de menor cuantía que en el varón, justifica en gran medida, que la Osteoporosis sea mucho más frecuente en el sexo femenino.

En nuestro trabajo, trataremos de exponer cómo la OPMG puede ser un instrumento muy valioso para un diagnóstico precoz de la osteoporosis pues sabemos que esta patología afecta al hueso alveolar maxilar y al hueso alveolar mandibular.

La OPMG o radiografía panorámica es una técnica radiológica que representa, en una única película, una imagen general de los maxilares, la mandíbula y los dientes, por tanto, es de primordial utilidad en el área dento-maxilo-mandibular. Los odontólogos pueden remitir a las mujeres al ginecólogo para una evaluación de la osteoporosis (3).

\section{La osteoporosis}

La Organización Mundial de la Salud define la osteoporosis como una enfermedad sistémica caracteri- zada por una masa ósea baja y un deterioro de la microarquitectura del tejido óseo, que conducen a una mayor debilidad ósea y a un aumento del riesgo de fracturas. Esta definición implica nuevos conceptos: por una parte, permite observar la enfermedad sin la existencia obligatoria de fracturas, y por otra, habla ya de la alteración de la microarquitectura (4).

En el Osteoporosis Consensus Development, celebrado en 1993, un grupo de expertos planteó una definición más completa que introduce un concepto relativo a la calidad ósea (microestructura) (5).

En 2001, La Conferencia de Consenso, patrocinada por los "National Institutes of Health (NIH)" de Estados Unidos, propuso una definición que pretende subrayar que la Osteoporosis es un trastorno cuantitativo y también cualitativo; en esta definición aparece el concepto de resistencia ósea (6).

Esta definición integra, por tanto, dos características: la cantidad de masa ósea (componente cuantitativo) y el concepto resistencia-fragilidad ósea (componente cualitativo).

Se tiende a equiparar la resistencia ósea con la densidad mineral ósea (DMO) puesto que la disminución de la misma se asocia fuertemente a la aparición de fracturas $(7,8)$. Pero también radica en este aspecto la explicación de las diferencias en la incidencia de fracturas en pacientes con la misma DMO (9).

El concepto de calidad ósea incluye un conjunto de variables estructurales del esqueleto no determinables mediante densitometría (DEXA) y que influyen en su resistencia al impacto. Junto a aspectos puramente cualitativos como los referentes a las características propias del tejido óseo, engloba otros como las características estructurales y geométricas del hueso (10).

\section{Epidemiología}

La osteoporosis es una enfermedad muy frecuente. Cerca de 3 millones de personas la padecen en España, la mayor parte de las cuales son mujeres. Aproximadamente, 30 de cada 100 mujeres la sufren después de la menopausia, llegando esta proporción al $52 \%$ después de los 65 años, aunque en ella debemos 
incluir a aquellas que padecen osteoporosis involutiva, no estrictamente relacionada con el climaterio (11).

\section{Etiología}

La osteoporosis tiene una etiología multifactorial y no del todo bien conocida. En el caso de las mujeres postmenopáusicas, la causa principal de la osteoporosis sería la falta de estrógenos. Se han descrito una serie de factores de riesgo que pueden contribuir al desarrollo de una osteoporosis (12).

El punto central en el diagnóstico de osteoporosis es la absorciometría dual de rayos X (DXA) de medida junto con los procedimientos de imágenes múltiples en radiología y otros químicos clínicos, de laboratorio y los resultados de biópsicos (13).

\section{Pronóstico}

Las variables más importantes para el pronóstico de la evolución de la enfermedad en los pacientes son la edad, el número de puntos terminales/periferia, periferia/zona trabecular, la densidad radiológica y el índice de masa ósea. Se puede utilizar una combinación de factores clínicos y radiográficos para identificar a los individuos con baja densidad mineral ósea (14).

Con el objeto de arrojar luz acerca de las posibilidades de diagnosticar la osteoporosis mediante OPMG se ha realizado la presente revisión bibliográfica utilizando las bases de datos Pub Med y Med Line. En el buscador se introdujeron las palabras "osteoporosis" y "ortopantomografía" obteniéndose un total de 124 entradas. Se acotó más la búsqueda, realizando una selección manual para escoger los artículos que pudieran ser útiles y se ordenaron las entradas obtenidas de una manera cronológica.

\section{DETERMINACIÓN DE LA OSTEOPOROSIS MEDIANTE EL USO DE ORTOPANTOMOGRAFÍAS}

Se ha considerado que utilizando la OPMG se obtienen índices fiables. Existen numerosos estudios que analizan los hallazgos radiográficos en las OPMG y radiografías periapicales, correlacionándolos con el diagnóstico precoz de osteoporosis, destacando la importancia del dentista en el diagnóstico precoz de esta enfermedad $(15,16)$. Esto es así puesto que la OPMG es una prueba rutinaria en la práctica odontológica actual. Estos estudios suelen basarse en la relación entre la osteoporosis y la reabsorción de la cresta residual de la mandíbula, reabsorción que también se asocia con la pérdida de hueso periodontal y la pérdida de dientes.

De los primeros autores que tomaron conciencia de la importancia de las OPMG orales como factor predictivo de la osteoporosis fueron Renner y cols.; examinaron a 11 mujeres postmenopáusicas, mediante la realización de OPMG y observaron que, de las 11 mujeres, las seis que estaban diagnosticadas de osteoporosis presentaban una mayor reabsorción de las crestas residuales que las que no tenían osteoporosis (17).

Con respecto a los índices para medir la pérdida de hueso alveolar en pacientes con osteoporosis, éstos fueron descritos por Bjorn y cols. (18). Estos autores medían la altura del nivel óseo en las zonas mesial y distal de los premolares y molares mandibulares y elaboraban una medida de la pérdida de hueso; este índice sólo se aplicó para diagnosticar periodontitis pero no osteoporosis. Se le llamó "Índice Roentgenográfico" y se le considera el primer índice que mide la pérdida de hueso mandibular (18).

Fue en marzo de 1991 cuando Benson y cols. desarrollaron el Panoramic Mandibular Index (PMI), que constituye un importante paso a la hora de analizar el nivel de osteoporosis. En este estudio se analizaron 353 radiografías y se aplicó el mencionado PMI, que consiste en dividir la anchura cortical mandibular del lado derecho de la OPMG entre la altura que va desde el borde inferior y superior del agujero mentoniano al borde inferior de la mandíbula, medido sobre una recta que pasa por el centro del agujero mentoniano y es perpendicular a la tangente del borde inferior del cuerpo mandibular. La medida que se tiene en cuenta es la media entre las dos mediciones; en el estudio, los autores observaron, al comparar distintos grupos de población, que las que tenían un PMI más bajo y por tanto mayor osteoporosis, eran las mujeres blancas de más de 70 años, que lo tenían por debajo de 0,3 (19). 
Más tarde, en 1993, Klemetti y cols. compararon el PMI con densitometrías en 355 mujeres postmenopáusicas en Finlandia y se concluyó que era muy difícil establecer una relación positiva entre dicho índice PMI y el estatus mineral óseo de las pacientes, medido mediante densitometrías, pero que, sin embargo, era de gran utilidad para prevenir la osteoporosis postmenopáusica, ya que si se observa un índice, en una paciente, inferior al de la media de la población en la que vive, se debe remitir esta paciente al reumatólogo para que sea tratada y diagnosticada de una posible osteoporosis (20).

Un año después, estos mismos autores, sobre esas 353 OPMG en las que habían medido el PMI, midieron la altura de la cortical mandibular y establecieron una clasificación ordinal para ordenar la forma de la cortical mandibular en tres grupos:

C1: el margen cortical inferior es lineal y grueso en ambos lados (cortical normal). C2: El margen cortical inferior presenta defectos semilunares de reabsorción lacunar y/o defectos endoóseos en la cortical y se ve también una leve erosión de la cortical en al menos un lado. C3: La cortical presenta grandes áreas de reabsorción lacunar, grandes defectos endoóseos, la cortical es claramente porosa y la corteza está severamente erosionada.

Los autores concluyen que no se debe utilizar una OPMG para diagnosticar osteoporosis, si bien su clasificación ordinal es bastante exacta con respecto a la osteoporosis.

En el 96, Taguchi y cols. realizan un estudio de casos y controles en 29 mujeres premenopáusicas y en 95 mujeres postmenopáusicas con osteoporosis. Las sometió a una densitometría para ver su status mineral óseo y se analizó la anchura y la forma mandibular (reabsorción); concluyeron que el uso de OPMG es válido para realizar un diagnóstico de sospecha de osteoporosis (21).

En el 98, Horner y cols. midieron, en la OPMG, la calidad ósea I-IV y el Mandibular Cortical Index (MCI) y realizaron densitometrías a 40 mujeres postmenopáusicas; se encontró una relación estadísticamente significativa entre Bone Quality Index (BQI) y densidad mineral ósea (BMD), siendo la asociación más poderosa la establecida entre $\mathrm{MCI}$ y $\mathrm{BMD}$, aunque la reproductibilidad del $\mathrm{MCI}$ es algo compleja (22).

El mismo año, estos mismos autores compararon las mediciones densitométricas y lineares (anchura cortical mandibular) y PMI de una TAC mandibular con la BMD de una densitometría de la mandíbula. En las 40 mujeres postmenopáusicas estudiadas, las medidas densitométricas del TAC no se correspondían con el densitómetro, pero la BMD sí que se relacionaba positivamente con la anchura cortical mandibular y el PMI, por lo que ambos índices pueden ser utilizados como indicadores de la densidad ósea mandibular (23).

En 1999, Jowitt y cols. realizaron un estudio para observar la reproductibilidad del $\mathrm{MCI}$ con observadores poco entrenados. 45 estudiantes de Odontología y también 4 expertos, analizaron 30 OPMG para hallar el MCI. El nivel de acuerdo entre los expertos fue bastante alto y se utilizó como "Gold Standard"; sin embargo, apenas hubo acuerdo entre los estudiantes: por lo tanto, los dentistas que vayan a hallar el $\mathrm{MCl}$ en sus pacientes deben estar bien entrenados (24).

También en 1999, Ledgerton y cols. examinaron 500 ortopantomografías con 5 índices, Gonial Index (GI), Mental Index( MI), PMI, MCI y Antegonial Index (AI). Los índices fueron examinados con respecto a la aplicación, reproductibilidad, edad, dentición y clase social. El GI, AI, PMI, MI no estaban relacionados con la edad pero sí el MCI; la dentición estaba relacionada con el PMI y MI. La clase social no influyó y la reproductibilidad fue aceptable en todos, menos en GI. Los cambios en el $\mathrm{MCI}$ con respecto a la edad van bien para diagnosticar la osteopenia; sin embargo, el fallo en la reproductibilidad del GI desaconseja su uso (25).

Devlin y cols., en 2002, realizaron un estudio para demostrar la validez de los índices mandibulares en el diagnóstico de una densidad mineral ósea reducida; 74 mujeres fueron sometidas a densitometría ósea de cadera, de columna (LS) y de muñeca. A las 74 mujeres se las sometió a una OPMG y se midió el AI, GI y MI. Sólo el MI fue indicativo a la hora de diferenciar pacientes de densidad mineral ósea alta y baja; sin embargo, se concluye que el solo uso de la OPMG no parece ser suficiente para diagnosti- 
car la osteoporosis, si bien puede ser integrada en un plan global para diagnosticarla con otros métodos (26).

También en 2002, Zlataric y cols. pretenden analizar distintos índices mandibulares en portadores de prótesis completa. Se analizaron 136 radiografías panorámicas en las cuales se analizaron $\mathrm{AI}, \mathrm{GI}, \mathrm{MI}, \mathrm{PMI}$ y MCI. AI, GI, MI y PMI iban descendiendo paulatinamente a medida que se avanzaba en edad. En cuanto al MCI sólo hubo C2 y C3, siendo el C3 más prevalente de una manera significativa en mujeres mayores de 75 años, por mayor prevalencia de osteoporosis, lo cual lo identifica como un índice válido (27).

En el mismo año, Zlataric y cols. publicaron un estudio cuyo fin era determinar si la densidad mineral ósea de la mandíbula y el GI, AI, MI y MCI están correlacionados con el índice de masa corporal (IMC). Estos índices se midieron en 136 OPMG. Las pacientes C3 de MCI tenían una BMD y IMC significativamente menores que las de C2; por lo tanto, pacientes con mayor BMD suelen tener menores medidas en índices mandibulares (28).

También en 2002, Horner K y cols. realizaron un estudio para determinar la utilidad de los índices radiográficos mandibulares en 135 mujeres sanas postmenopáusicas; la BMD fue medida mediante una densitometría de cadera y espinal y un TAC mandibular para hallar el MI y se halló igualmente el IMC. Un MI menor de $3 \mathrm{~mm}$ se asocia con osteoporosis y un BMI bajo puede conducir más fácilmente a padecer osteoporosis (29).

En 2003, Zlataric y cols. realizaron un estudio en el que, sobre 136 ortopantomografías (40 hombres y 96 mujeres) se hizo el MCI y un análisis de las ortopantomografías para hallar la densidad mineral ósea mediante una medición densitométrica de la mandíbula; se halló que el grado C3 del MCI está íntimamente relacionado con una baja BMD de manera significativa. El acuerdo entre los cuatro observadores que analizaron las OPMG fue total (30).

En 2005, Halling y cols. llevaron a cabo un estudio para determinar la validez del índice de Klemetti MCI para diagnosticar la osteoporosis y la osteopenia; para ello se hicieron 211 OPMG y también 211 DEXAS de talón y se concluyó que había más osteopénicos y osteoporóticos en C3 que en C2 y C1. Por lo tanto, el estudio determinó que si la ortopantomografía de un paciente no se incluye en el grupo C3, tiene muchas probabilidades, de una manera estadísticamente significativa, de no estar padeciendo osteoporosis ni osteopenia (31).

En 2005 igualmente, Dutra y cols. buscaron evaluar el MI y el AI. Se evaluaron 312 radiografías panorámicas. Tanto el MI como el AI fueron significativamente menores en los dos grupos de mujeres mayores al compararlos con los de los hombres. Se concluyó que la pérdida de densidad y volumen en la cortical mandibular estaba influenciada por la edad y el sexo. Dos observadores probaron que el estudio de MI era una técnica reproducible (32).

En ese mismo año, Hüpsch-Marzec y cols. realizaron un estudio de casos-controles en el que los casos son mujeres que han sufrido una fractura osteoporótica en el radio, entre 51 y 69 años y los controles, mujeres sanas; se les realizaron ortopantomografías donde se midió MCI y PMI y se vio que entre los casos y los controles había diferencias estadísticamente significativas en lo referente a $\mathrm{C} 3$ de $\mathrm{MCI}$ pero no a C2 y C1 ni al PMI; por lo tanto, el grado C3 de $\mathrm{MCI}$ es un gran indicador de osteoporosis (33).

También en 2006, Dutra y cols. realizaron un estudio para evaluar los cambios morfológicos de la mandíbula en osteoporóticas y osteopénicas postmenopáusicas y edéntulas; 52 pacientes fueron sometidas a OPMG y DEXA lumbar y femoral; se midió el GI, AI y MI. AI y MI fueron estadísticamente significativos más pequeños en el grupo de osteopénicas y osteoporóticas que en el normal. También se vio que la delgadez en la cortical mandibular está influenciada por la edad (34).

En 2007, Devlin y cols. hicieron un estudio para determinar la eficacia del MCI y del mandibular Cortical Width (MCW) como predictores de la osteoporosis. Se encontró que aquellas mujeres dentro del grupo C3 de $\mathrm{MCl}$ eran más propensas a osteoporosis de una manera significativa y que si en el MCW la cortical era inferior a $3 \mathrm{~mm}$ debería estudiarse si la paciente padece o no osteoporosis postmenopáusica (35). 
En 2007 también, Dutra y cols. buscaron ver si realmente el MI servía para validar la osteoporosis; para ello se sirvió de diez mandíbulas disecadas y concluyó que el MI se podría utilizar para medir el status óseo de una persona, tras eliminar la magnificación de las ortopantomografías (36).

Igualmente en 2007, Vlasiadis y cols. publicaron un estudio cuyo objetivo era evaluar la validez de MCW, MCI, PMI y el grado de reabsorción de la cresta alveolar como predictores de osteoporosis. A 133 mujeres postmenopáusicas de entre 38 y 80 años, se les realizó una OPMG para medir $\mathrm{MCW}, \mathrm{MCI}$, PMI y grado de reabsorción de la cresta alveolar, así como una DEXA ósea lumbar. Se vio que las osteoporóticas y osteopénicas tenían una disminución de $1 \mathrm{~mm}$ en el MCW con respecto a las normales, de una manera significativa. Los grados C2 y C3 aparecían de una manera significativa más frecuentemente en mujeres osteopénicas y osteoporóticas; por lo tanto, los índices radiomorfométricos mandibulares pueden ser útiles en el pronto diagnóstico de osteoporosis (37).

En ese mismo año de 2007, Uysal y cols. buscaron determinar el efecto del sexo en $\mathrm{MCI}$. C1 y C3 tuvieron mayor presencia, de una manera significativa, en mujeres que en hombres. C3 fue mayor, de forma significativa, en mujeres de más de 60 años; por lo tanto las mujeres más mayores tienen mayor osteoporosis según $\mathrm{MCl}$ que los hombres, de modo que la edad y el sexo influyen en el MCI. (38).

En 2008, Gulsahi y cols. publicaron un estudio en el que se analizaron 1863 OPMG. En comparación con el grupo de 20-49 años, la probabilidad de C3 en MCI en mayores de 70 años y 50-69 años fue 9 veces mayor y más significativa; la probabilidad de C3 en pacientes desdentados fue 2,68 veces mayor que en pacientes parcialmente desdentados. C3 también fue más frecuente, de manera significativa en mujeres mayores de 70 y 50-69 que en hombres; C3 fue más frecuente en $\mathrm{MI}$ menor de $3 \mathrm{~mm}$ que en $\mathrm{MI}$ mayor de $3 \mathrm{~mm}$, la probabilidad de C3 en PMI menor de 0,30 fue 9,78 veces mayor, de un modo significativo, que en PMI mayor de 0,30.

Por lo tanto, independientemente del sexo, pacientes con C3 de MCI, MI menor de 3 mm y PMI menor de 0,30 , deben ser considerados en riesgo de padecer osteoporosis (39).

También en 2008, en un estudio de Çakur y cols. se trató de ver si el MCI es un buen predictor de osteoporosis; para ello, se hacen ortopantomografías en 25 mujeres con osteoporosis postmenopáusicas, se mide el $\mathrm{MCI}$, se hace una medición de densidad ósea en la mandíbula y se somete a las pacientes a densitometría ósea lumbar. Hubo una relación significativa entre el MCI y la densitometría ósea lumbar. Por lo tanto, el MCI puede ser un buen método para la detección de osteoporosis en gabinete dental (40).

Ya en 2010, Kiswanjaya y cols., en un estudio, buscaron relacionar el MCI y la rigidez o fragilidad ósea en pacientes japoneses mayores. El estudio incluyó 519 personas sanas, en mujeres se encontraron diferencias significativas entre C1 y C3, y C2 у C3, siendo mucho más frágil, de una manera significativa el grado C3. Por lo tanto, se concluye que el $\mathrm{MCl}$ puede ser útil para un diagnóstico precoz de la osteoporosis si remitimos al reumatólogo a los pacientes que inducen a sospechar la existencia de osteoporosis (41).

También en 2010, Leite y cols. buscaron relacionar siete índices (MCI, anchura visual de cortical mandibular, MI, AI, Antegonial depth, gonial angle y antegonial angle) con la MBD, medida por medio de densitometría lumbar en 351 mujeres; se concluyó que el MI, el MCI y una estimación visual de la anchura de la cortical mandibular inferior son los índices más precisos a la hora de poder diagnosticar precozmente la osteoporosis postmenopáusica (42).

Ese mismo año de 2010, Mudda y cols. publicaron un estudio cuyo objetivo fue evaluar los cambios en el hueso mandibular en mujeres pre y postmenopáusicas usando los MCI, MI y PMI. Se hicieron ortopantomografías a 60 mujeres y se dividieron entre pre y postmenopáusicas. Se calculó el MCI, MI y PMI. MCI, MI y PMI están relacionados con la osteoporosis postmenopáusica. Pacientes con C3 del MCI sólo se vieron en el grupo postmenopáusico de más de 54 años; las medidas del PMI y MI fueron más altas de una manera estadísticamente significativa en el grupo premenopáusico. El PMI se relacionó positiva- 
mente con la edad. Estos índices deben ser usados por los dentistas para diagnosticar a mujeres con alto riesgo de osteoporosis (43).

En 2010 igualmente, Marandi y cols., en su estudio, se plantearon como objetivo evaluar la eficacia de MI, PMI y MCI como indicadores de osteoporosis y compararlos con densitometrías óseas lumbares y de cadera. 67 mujeres mayores de 35 años fueron sometidas a estas dos densitometrías y a ortopantomografía. Hubo diferencias estadísticamente significativas al comparar la BMD entre $\mathrm{C} 1$ y C3 del $\mathrm{MCI}$; en el PMI no hubo diferencias estadísticamente significativas, pero MI sí tuvo esas diferencias entre el grupo normal y el osteopénico. MI y $\mathrm{MCI}$ pueden ayudar a diagnosticar precozmente a una paciente en riesgo de osteoporosis (44).

Javelaviciene y cols., en 2010, hicieron un estudio que buscaba determinar la relación entre la BMD calcánea medida con una densitometría de cadera y el MCI, MI y PMI medidos mediante OPMG en mujeres postmenopáusicas. Se estudiaron 129 postmenopáusicas que se sometieron a OPMG sobre las que se calcularon MI y PMI. Se encontraron diferencias estadísticamente significativas entre el grupo normal y osteopénicas y osteoporóticas, tanto para MI como PMI; por tanto, se pueden utilizar tanto PMI como MI para identificar a pacientes con baja densidad ósea general (45).

Gulsahi y cols., en 2010, realizaron un estudio que evaluó la BMD de mandíbula, maxilar y cadera, mediante densitometría ósea y los comparó entre sí y con $\mathrm{MCI}$, PMI y MI; MCI, PMI y MI no se relacionaron con la BMD ni maxilar ni mandibular, aunque la muestra estudiada no era muy significativa ,18 hombres y 31 mujeres (46).

Dagistan y cols., en 2010 igualmente, en su estudio, buscaron comparar el AI, MI, PMI y MCI de 20 hombres sin patología y 20 con osteoporosis, usando ortopantomografías. No se encontraron diferencias estadísticamente significativas entre casos y controles en lo referente al MCI. MI, AI y PMI pueden ser utilizados como un método auxiliar para el diagnóstico de osteoporosis en hombres, habiendo ya demostrado que resultan de gran utilidad en mujeres con osteoporosis. Sin embargo, el $\mathrm{MCl}$ que resulta de gran ayuda en mujeres, en los hombres no ha resultado útil, aunque se necesitan más estudios con más pacientes (47).

Lin y cols., en 2010, realizaron un estudio para investigar los cambios en la densidad mineral ósea de pacientes seniles con osteoporosis en la mandíbula y comparar la pérdida ósea mandibular con la sistémica. Los ancianos con osteoporosis tenían menor MCW y PMI de manera significativa que los ancianos sanos. La densidad ósea alveolar y la altura ósea alveolar fueron menores de manera significativa en ancianos sanos que en jóvenes sanos; por lo tanto, los ancianos con osteoporosis tienen una pérdida severa y significativa de hueso alveolar (48).

Taguchi publicó en 2010 un artículo en el que insiste en la idea de que muchos pacientes con osteoporosis no se diagnostican porque normalmente no hay síntomas presentes antes de una fractura. Las clínicas dentales pueden ofrecer una forma nueva de detección. Por ejemplo, la anchura cortical y forma de la mandíbula detectadas en las radiografías panorámicas pueden ser índices apropiados para detectar a los individuos con osteoporosis. Hasta la fecha, varios investigadores han demostrado una asociación significativa entre los índices corticales en las radiografías panorámicas y la densidad mineral ósea del esqueleto en general. Además, en dos ensayos clínicos recientes japoneses, aproximadamente el $95 \%$ de las mujeres que fueron identificadas por los dentistas en sus clínicas, por medio de los estudios corticales, tenían osteopenia u osteoporosis. Estos hallazgos apoyan la posibilidad de que las clínicas dentales pueden ofrecer una plataforma de nueva clasificación para identificar a individuos con osteoporosis que de lo contrario, quedarían sin ser detectados (49).

En 2011, Hastar y cols. se plantearon como objetivo evaluar la influencia del sexo y estado dental en el MI, PMI y MCI de radiografías panorámicas en ancianos con y sin osteoporosis. 487 pacientes entre $60 \mathrm{y}$ 88 años, 239 hombres y 248 mujeres se someten a ortopantomografías para hallar los índices. Entre hombres y mujeres hubo diferencias significativas en MCW y PMI (menores en mujeres sobre todo en mayores de 60 años). MCW y PMI fueron más bajos en edéntulos que en el resto. MCI: C1 fueron más 
frecuentes en hombres, de manera significativa, C2 más frecuente en mujeres y C3 se halló sólo en mujeres. C1 fue más frecuente en pacientes sin osteoporosis de una manera significativa. C2 fue más frecuente en osteoporóticos. De forma significativa, los pacientes con osteoporosis tenían más bajo el MCW y el PMI que los no osteoporóticos; los osteoporóticos estaban incluidos en el grupo de desdentados de manera estadísticamente significativa.

Por lo tanto, este estudio muestra que existen diferencias estadísticamente significativas, según el sexo y el status dental en el $\mathrm{MCI}, \mathrm{MCW}$ y PMI entre los pacientes con y sin osteoporosis (50).

También en 2011 López-López y cols. realizaron una búsqueda en la base de datos Medline Pubmed, con el fin de identificar aquellos artículos que tienen que ver con la asociación entre los resultados de los rayos X observados en las OPMG y el diagnóstico de la osteoporosis, así como aquellos que estudian la densidad mineral ósea de la mandíbula. Casi todos los artículos indican que, al examinar las radiografías orales, es posible detectar los signos indicativos de la osteoporosis. Los autores concluyen que la combinación de los índices de la mandíbula, junto con los estudios sobre el riesgo de fractura, pueden ser útiles como indicadores de diagnóstico precoz de la osteoporosis. Los índices visuales y morfométricos parecen ser especialmente importantes en las OPMG. Por lo tanto, López-López y cols. concluyen que existe evidencia científica de que la disminución de la masa ósea por la osteoporosis también afecta a la mandíbula y que esta pérdida de densidad ósea puede ser evidenciada en las exploraciones orales rutinarias o previas a tratamientos dentales, tanto en ortopantomografías como en radiografías periapicales (51).

\section{CONCLUSIONES}

1. El estudio de algunos índices mandibulares valorados en ortopantomografías puede servir para detectar precozmente la osteoporosis. Y remitir a los pacientes a los especialistas para tratar dicha enfermedad.

2. De todos los índices radiomorfométricos mandibulares parece ser que el MCI es el que presenta una mayor eficacia, seguido por el MI y PMI.
3. Otros índices como el AI y el GI presentan una muy baja eficacia y no deberían ser empleados.

\section{BIBLIOGRAFÍA}

1. Albright F, Smith PH, Richardson AM. Postmenopausal osteoporosis. JAMA 1941;116:24.

2. Dempster DW, Lindsay R. Pathogenesis of osteoporosis. Lancet 1993;341:260-262:65-74.

3. Friedlander $\mathrm{AH}$. The physiology, medical management and oral implications of menopause. J Am Dent Assoc 2002;133(1):73-81.

4. Copenhague-Consensus Development Conference. Am J Med 1991;90:107-10.

5. Consensus Development Conference: diagnosis, prophylaxis and treatment of osteoporosis. Am J Med 1993;94:646-50.

6. NIH Consensus development Panel on Osteoporosis Prevention, Diagnosis and Therapy. JAMA 2001;285:785-95

7. Sosa M, Gómez de Tejada MJ, Hernández D. Prevención de la osteoporosis. Concepto, clasificación, factores de riesgo y clínica de la osteoporosis. Rev Esp Enferm Metab Óseas 2001;10(Supl A):7-11.

8. Marsall D, Johnell O, Wedel H. Meta-analysis of how well measures of bone mineral dendity predict occurrence of osteoporotic fractures. $\mathrm{Br}$ Med J 1996;312:1254-1259.

9. Hermoso de Mendoza MT. Classification of osteoporosis. Risk factors. Clinical manifestations and differential diagnosis. An Sist Sanit Navar 2003; 26 Suppl 3:29-52.

10. Seeman E, Delmas D. Bone quality, the material and structural basis of bone strength and fragility. N Engl J Med 2006;354:2250-61.

11. Bączyk G, Opala T, Kleka P. Quality of life in postmenopausal women with reduced bone mi- 
neral density: psychometric evaluation of the Polish version of QUALEFFO-41. Arch Med Sci 2011;7(3):476-85.

12. Yazici S, Korkmaz U, Erkan M, Korkmaz N, Erdem Baki A, Alçelik A, et al. The effect of breastfeeding duration on bone mineral density in postmenopausal Turkish women: a population-based study. Arch Med Sci 2011;7(3):486-92.

13. Bartl C, Bartl R. Secondary osteoporosis: Pathogenesis, types, diagnostics and therapy. Radiologe 2011;51(4):307-324.

14. Licks R, Licks V, Ourique F, Radke Bittencourt H, Fontanella V Development of a prediction tool for low bone mass based on clinical data and periapical radiography. Dentomaxillofac Radiol 2010;39(4):224-30.

15. Çakur B, Dagistan S, Sahin A, Harorli A, Yilmaz A. Reliability of mandibular cortical index and mandibular bone mineral density in the detection of osteoporotic women Dentomaxillofac Radiol 2009;38:255-61.

16. Vlasiadis KZ, Skouteris CA, Velegrakis GA, Fragouli I, Neratzoulakis JM, Damilakis J et al. Mandibular radiomorphometric measurements as indicators of possible osteoporosis in postmenopausal women. Maturitas 2007;20;58:226-35.

17. Renner RP, Boucher LJ, Kaufman HW. Osteoporosis in postmenopausal women. J Prosthet Dent 1984;52:581-8.

18. Björn AL, Björn H, Halling A. An abbreviated index for periodontal bone height. Odontol Revy 1975; 26:225-30.

19. Benson BW, Prihoda TJ, Glass BJ. Variations in adult cortical bone mass as measured by a panoramic mandibular index. Oral Surg Oral Med Oral Pathol 1991;71:349-56.

20. Klemetti E, Kolmakov S, Heiskanen P, Vainio P, Lassila V. Panoramic mandibular index and bone mineral densities in postmenopausal women.Oral Surg Oral Med Oral Pathol 1993;75:774-9.
21. Taguchi A, Suei Y, Ohtsuka M, Otani K, Tanimoto $\mathrm{K}$, Ohtaki $M$. Usefulness of panoramic radiography in the diagnosis of postmenopausal osteoporosis in women. Width and morphology of inferior cortex of the mandible. Dentomaxillofac Radiol 1996;25: 263-7.

22. Horner K, Devlin H. The relationships between two indices of mandibular bone quality and bone mineral density measured by dual energy X-ray absorptiometry. Dentomaxillofac Radiol 1998; 27:17-21.

23. Horner K, Devlin H. The relationship between mandibular bone mineral density and panoramic radiographic measurements. J Dent 1998;26:337-43.

24. Jowitt N, MacFarlane T, Devlin H, Klemetti E, Horner K. The reproducibility of the mandibular cortical index. Dentomaxillofac Radiol 1999;28: 141-4.

25. Ledgerton D, Horner K, Devlin H, Worthington $\mathrm{H}$. Radiomorphometric indices of the mandible in a British female population. Dentomaxillofac Radiol 1999;28:173-81.

26. Devlin H, Horner K. Mandibular radiomorphometric indices in the diagnosis of reduced skeletal bone mineral density. Osteoporos Int 2002;13: 373-8.

27. Zlatarić DK, Celebić A, Lazić B, Baucić I, Komar $D$, Stipetić-Ovcaricek $J$ et al. Influence of age and gender on radiomorphometric indices of the mandible in removable denture wearers. Coll Antropol 2002;26:259-66.

28. Zlatarić DK, Celebić A, Kobler P. Relationship between body mass index and local quality of mandibular bone structure in elderly individuals. J Gerontol A Biol Sci Med Sci 2002;57(9):M588-93.

29. Horner K, Devlin H, Harvey L. Detecting patients with low skeletalbone mass. J Dent 2002;30:1715.

30. Zlatarić DK, Celebić A. Clinical bone densitometric evaluation of the mandible in removable denture 
wearers dependent on the morphology of the mandibular cortex. J Prosthet Dent 2003;90:86-91.

31. Halling A, Persson GR, Berglund J, Johansson $\mathrm{O}$, Renvert S. Comparison between the Klemetti index and heel DXA BMD measurements in the diagnosis of reduced skeletal bone mineral density in the elderly. Osteoporos Int 2005;16:999-1003.

32. Dutra V, Yang J, Devlin H, Susin C. Radiomorphometric indices and their relation to gender, age, and dental status. Oral Surg Oral Med Oral Pathol Oral Radiol Endod 2005;99:479-84.

33. Hüpsch-Marzec H, Ilewicz L, Klima D, Swietochowska E, Pluskiewicz W, Mykietów B. Assessment of selected parameters measured on panoramic radiographs in postmenopausal women. Przegl Lek 2005;62:1387-9.

34. Dutra V, Devlin H, Susin C, Yang J, Horner K, Fernandes AR. Mandibular morphological changes in low bone mass edentulous females: evaluation of panoramic radiographs. Oral Surg Oral Med Oral Pathol Oral Radiol Endod 2006; 102:663-8.

35. Devlin H, Karayianni K, Mitsea A, Jacobs R, Lindh C, Van der Stelt P et al. Diagnosing osteoporosis by using dental panoramic radiographs: the OSTEODENT project. Oral Surg Oral Med Oral Pathol Oral Radiol Endod 2007;104:821-8.

36. Dutra V, Susin C, da Costa NP, Veeck EB, Bahlis A, Fernandes Ada R. Measuring cortical thickness on panoramic radiographs: a validation study of the Mental Index. Oral Surg Oral Med Oral Pathol Oral Radiol Endod 2007;104:686-91.

37. Vlasiadis KZ, Skouteris CA, Velegrakis GA, Fragouli I, Neratzoulakis JM, Damilakis J et al. Mandibular radiomorphometric measurements as indicators of possible osteoporosis in postmenopausal women. Maturitas 2007;20(58):226-35.

38. Uysal S, Cağirankaya BL, Hatipoğlu MG. Do gender and torus mandibularis affect mandibular cortical index? A cross-sectional study. Head Face Med 2007;30(3):37.
39. Gulsahi A, Yüzügüllü B, Imirzalioglu P, Genç Y. Assessment of panoramic radiomorphometric indices in Turkish patients of different age groups, gender and dental status. Dentomaxillofac Radiol 2008;37:288-92.

40. Çakur B, Sahin A, Dagistan S, Altun O, Caglayan F, Miloglu $O$ et al. Dental panoramic radiography in the diagnosis of osteoporosis. J Int Med Res 2008; 36:792-9.

41. Kiswanjaya B, Yoshihara A, Deguchi T, Hanada N, Miyazaki H. Relationship between the mandibular inferior cortex and bone stiffness in elderly Japanese people. Osteoporos Int 2010;21:433-8.

42. Leite AF, Figueiredo PT, Guia CM, Melo NS, de Paula AP. Correlations between seven panoramic radiomorphometric indices and bone mineral density in postmenopausal women. Oral Surg Oral Med Oral Pathol Oral Radiol Endod 2010; 109:449-56.

43. Mudda JA, Bajaj M, Patil VA. A Radiographic comparison of mandibular bone quality in pre- and post-menopausal women in Indian population. J Indian Soc Periodontol 2010;14:121-5.

44. Marandi S, Bagherpour A, Imanimoghaddam M, Hatef M, Haghighi A. Panoramic-based mandibular indices and bone mineral density of femoral neck and lumbar vertebrae in women. J Dent (Tehran) 2010;7:98-106.

45. Jagelaviciene E, Kubilius R, Krasauskiene A. The relationship between panoramic radiomorphometric indices of the mandible and calcaneus bone mineral density. Medicina (Kaunas) 2010;46: 95-103.

46. Gulsahi A, Paksoy CS, Ozden S, Kucuk NO, Cebeci AR, Genc Y. Assessment of bone mineral density in the jaws and its relationship to radiomorphometric indices. Dentomaxillofac Radiol 2010;39:284-9.

47. Dagistan S, Bilge OM. Comparison of antegonial index, mental index, panoramic mandibular index and mandibular cortical index values in the panoramic radiographs of normal males and male 
patients with osteoporosis. Dentomaxillofac Radiol 2010;39:290-4.9.

48. Lin ZT, Wang TM, Ge JY, Lin H, Zhu XF. Analysis of mandibular bone mineral density of senile osteoporosis patients. Zhonghua Kou Qiang Yi Xue Za Zhi 2010;45:214-8.

49. Taguchi A. Triage screening for osteoporosis in dental clinics using panoramic radiographs. Oral Dis 2010;16(4):316-27.

50. Hastar E, Yilmaz HH, Orhan H. Evaluation of mental index, mandibular cortical index and panoramic mandibular index on dental panoramic radiographs in the elderly. Eur J Dent 2011;5:60-7.
51. López-López J, Estrugo-Devesa A, Jane-Salas E, Ayuso-Montero R, Gómez-Vaquero C. Early diagnosis of osteoporosis by means of orthopantomograms and oral x-rays: A systematic review. Med Oral Patol Oral Cir Bucal 2011;1(16):e905-13.

\section{CORRESPONDENCIA}

J. R. Corcuera Flores

Facultad de Odontología.

Universidad de Sevilla

Avicena, s/n

41009 Sevilla

Correo electrónico: jcorcuera@us.es 